CPHT-RR103.1111

LU TP 11-42

December 2011

\title{
Gauge invariance of DVCS off an arbitrary spin hadron: the deuteron target case
}

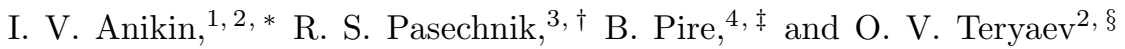 \\ ${ }^{1}$ Institute for Theoretical Physics, University of Regensburg, D-93040 Regensburg, Germany \\ ${ }^{2}$ Bogoliubov Laboratory of Theoretical Physics, JINR, 141980 Dubna, Russia \\ ${ }^{3}$ Department of Astronomy and Theoretical Physics, Lund University, SE 223-62 Lund, Sweden \\ ${ }^{4}$ CPHT, École Polytechnique, CNRS, 91128 Palaiseau, France
}

(Dated: November 9, 2018)

\begin{abstract}
We study the deeply virtual Compton scattering off a spin-one particle, as the case for the coherent scattering off a deuteron target. We extend our approach, formulated initially for a spinless case, and discuss the role of twist three contributions for restoring the gauge invariance of the amplitude. Using twist three contributions and relations, which emanate from the QCD equations of motion, we derive the gauge invariant amplitude for the deeply virtual Compton scattering (DVCS) off hadrons with spin 1. Using the derived gauge invariant amplitude, the single spin asymmetry is discussed.
\end{abstract}

PACS numbers: 12.38.Bx, 13.60.Le

\section{INTRODUCTION}

Deeply virtual Compton scattering (DVCS) off the deuteron target has recently attracted much attention from the experimental point of view [1-4]. One of the main reasons of this interest is the fact that the DVCS process gives information about the generalized parton distributions (GPDs). From the theoretical point of view, the leading twist-2 GPDs for the deuteron were studied in 5-7]. However, the leading twist-2 accuracy for the DVCS amplitude, calculated in the case where the final deuteron gets a significant transverse momentum, is not enough for the study of such processes. This is due to the fact that in the essential case of sizable transverse transfer momentum, $\Delta_{T} \neq 0$, the leading twist-2 approximation, in the Bjorken limit, is not sufficient for the photon gauge invariance of the DVCS amplitude (see, for example, [8]) ${ }^{1}$. Besides, the relevant terms are proportional to the transverse component of the momentum transfer and provide the leading contribution to some observables.

Extending the Ellis-Furmanski-Petronzio-Efremov-Teryaev (EFPET) approach (see, 9, 10]) to the nonforward case, this problem was first solved in [11] where it was demonstrated how the inclusion of twist-3 contributions related to the matrix elements of quark-antiquark-gluon operators, can restore the gauge invariance of the DVCS amplitude off a (pseudo)scalar particle (e.g. pion, $\mathrm{He}^{4}$ ). Then, the main ideas of [11] were used and generalized for the nucleon target (see, [12-17] and [18] for different processes).

In this paper, we adhere to the approach [11] and make a comprehensive analysis of the twist three contributions to the DVCS amplitude off a spin- 1 hadron $^{2}$. Since the parameterizations of the relevant hadronic matrix elements of the quark-gluon operators depend much on the spin of the external hadrons, following the very useful idea of [12] we start our study with a parametrization-free approach, and then apply it to the specific case of spin-1 hadron.

\footnotetext{
*Electronic address: anikin@theor.jinr.ru, Igor.Anikin@physik.uni-regensburg.de

$\dagger$ Electronic address: Roman.Pasechnik@thep.lu.se

‡Electronic address: pire@cpht.polytechnique.fr

$\S$ Electronic address: teryaev@theor.jinr.ru

1 Throughout this paper, we deal with the $U(1)$ gauge invariance of the amplitude rather than the $S U(3)_{c}$ gauge invariance. Concerning the QCD gauge, we fix it by choosing the axial gauge for gluons, $A^{+}=0$.

2 Actually, the method described in [1] and in this paper is suitable for a study of DVCS off an arbitrary spin hadron.
} 

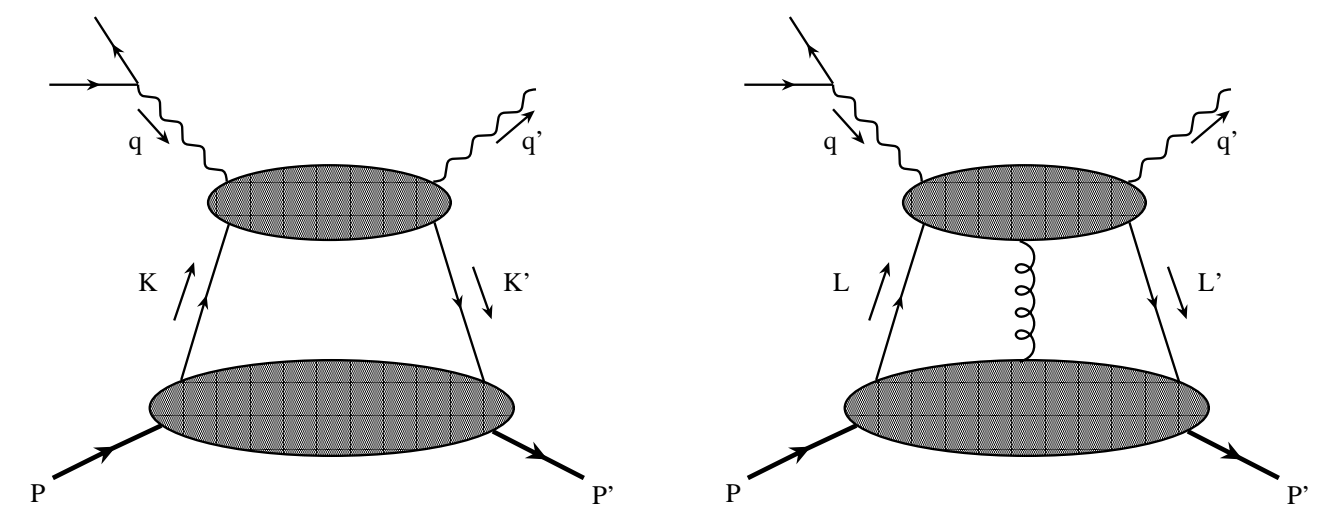

Figure 1: The Feynman diagrams corresponding to the deeply virtual Compton scattering. The DVCS amplitude with two-particle correlators is depicted on the left panel, while the amplitude with the three-particle correlators on the right panel. Notations: $P \equiv p_{1}, \quad P^{\prime} \equiv p_{2}, K \equiv k-\Delta / 2 \approx x P-\Delta / 2, \quad K^{\prime} \equiv k+\Delta / 2 \approx x P+\Delta / 2$, $L \equiv k_{1}-\Delta / 2 \approx x_{1} P-\Delta / 2, \quad L^{\prime} \equiv k_{2}+\Delta / 2 \approx x_{2} P+\Delta / 2$. Here, $k$ and $k_{i}$ correspond to the loop momenta in the diagrams.

\section{KINEMATICS AND APPROXIMATIONS}

Let us start with the discussion of the kinematics and approximations which we use in this paper. The process we consider is

$$
\gamma^{*}(q)+D\left(p_{1}\right) \rightarrow \gamma\left(q^{\prime}\right)+D\left(p_{2}\right) .
$$

Here, we mainly focus on the deuteron as a target but all our approach is suitable for any spin-one hadron target. At the Born level, the Feynman diagrams corresponding to the considered process are depicted in Fig. 11, This process is a hard exclusive reaction for which a QCD factorization theorem applies. In this case, the virtuality of the initial off-shell photon is used as the large scale, i.e. $q^{2}=-Q^{2} \rightarrow \infty$, while the final photon is on-shell with $q^{\prime 2}=0$. Besides, this asymptotical regime is identical to the light-cone formalism. Therefore, we first introduce a light-cone basis which is constructed by the "plus" and "minus" vectors:

$$
\begin{aligned}
& n^{\star}=\Lambda(1,0,0,1), \\
& n=\frac{1}{2 \Lambda}(1,0,0,-1), \\
& n^{\star} \cdot n=1,
\end{aligned}
$$

where $\Lambda$ is an arbitrary and dimensionful constant which can be expressed via the Lorentz invariants. The exact form of $\Lambda$ as a function of invariants depends on the frame which one works in.

In the present paper, we consider the DVCS amplitude up to the twist three accuracy, discarding the contributions associated with the twist four and higher. Such a constraint imposes the following relations for the hadron average and transfer momenta :

$$
\begin{aligned}
& P=\frac{p_{1}+p_{2}}{2}=n^{\star}+\frac{\bar{M}^{2}}{2} n \approx n^{\star}, \\
& \Delta=p_{2}-p_{1}=-2 \xi P+2 \xi \bar{M}^{2} n+\Delta^{T} \approx-2 \xi P+\Delta^{T}, \\
& P \cdot \Delta=0, \quad \Delta^{2}=t \approx 0 .
\end{aligned}
$$

Notice that keeping the $\bar{M}^{2}$-term in the Sudakov decomposition of the relative momentum $P$ (see, (3)) leads to the necessity to include the twist four contributions as well, which goes beyond the scope of the present paper. Since corrections of the order $O\left(\Delta_{T}^{2} / Q^{2}\right)$ demand a special care, at this moment, we postpone the study of them until a forthcoming paper. Notice that the detailed analysis of these contributions has recently been presented in [19, 20]. 
It is also instructive to introduce the photon average momentum:

$$
\begin{aligned}
& \bar{Q}=\frac{q+q^{\prime}}{2}=q-\frac{\Delta}{2}=q^{\prime}+\frac{\Delta}{2}, \quad q^{\prime}=\left(P \cdot q^{\prime}\right) n, \\
& \left(P \cdot q^{\prime}\right)=(P \cdot \bar{Q})=(P \cdot q) .
\end{aligned}
$$

One has to emphasize that the approximations discussed in this section do not affect the generality of our study and can be applied to a study of arbitrary spin hadrons.

\section{FACTORIZATION AND THE GAUGE INVARIANT AMPLITUDE}

In this section, we briefly describe the factorization procedure applied to the DVCS amplitude up to the twist three accuracy. The details of this factorization can be found in [11, 18, 21, 22].

At the Born level, the sum of the amplitudes with the two- and three-particle correlators, or $T_{\mu \nu}^{(1)}$ and $T_{\mu \nu}^{(2)}$ amplitudes, has the following form (for the DIS case, see [10]):

$$
\begin{aligned}
& T_{\mu \nu}^{(1)}+T_{\mu \nu}^{(2)}=\int d^{4} k \operatorname{tr}\left[E_{\mu \nu}(k) \Gamma(k)\right] \\
& +\int d^{4} k_{1} d^{4} k_{2} \operatorname{tr}\left[E_{\mu \rho \nu}\left(k_{1}, k_{2}\right) \Gamma^{\rho}\left(k_{1}, k_{2}\right)\right]
\end{aligned}
$$

where $E_{\mu \nu}$ and $E_{\mu \rho \nu}$ are the coefficient functions, at the Born approximation, with two quark legs and two quark and one gluon legs, respectively. In Eq. (5), we use the following notations:

$$
\begin{aligned}
& \Gamma_{\underline{\alpha \beta}}(k)=-\int_{-\infty}^{\infty} d^{4} z e^{i(k-\Delta / 2) z}\left\langle p_{2}\left|\psi_{\underline{\alpha}}(z) \bar{\psi}_{\underline{\beta}}(0)\right| p_{1}\right\rangle, \\
& \Gamma_{\underline{\alpha \beta}}^{\rho}\left(k_{1}, k_{2}\right)=-\int_{-\infty}^{\infty} d^{4} z_{1} d^{4} z_{2} e^{i\left(k_{1}-\Delta / 2\right) z_{1}+i\left(k_{2}-k_{1}\right) z_{2}} \\
& \times\left\langle p_{2}\left|\psi_{\underline{\alpha}}\left(z_{1}\right) g A^{\rho}\left(z_{2}\right) \bar{\psi}_{\underline{\beta}}(0)\right| p_{1}\right\rangle,
\end{aligned}
$$

where the underlined indices $\underline{\alpha}, \underline{\beta}$ denote the Dirac spin indices while the other indices correspond to the Lorentz ones.

It is convenient to choose the axial gauge condition for gluons, i.e. $n \cdot A=0$, where $n$ is the light-cone vector defined in Eq. (2). Then, we carry out a decomposition of the loop momentum $k$ over the light-cone vectors (the Sudakov decomposition) as follows

$$
k_{\mu}=x P_{\mu}+(k \cdot P) n_{\mu}+k_{\mu}^{T} \approx x P_{\mu}+k_{\mu}^{T},
$$

where $x=k \cdot n$. As the next step of the factorization procedure, we perform the replacement for the integration momentum in Eq. (5) as

$$
d^{4} k_{i} \rightarrow d^{4} k_{i} d x_{i} \delta\left(x_{i}-k_{i} \cdot n\right) .
$$

This allows us to expand the two-quark coefficient function $E_{\mu \nu}$ (see, (5) ) in a Taylor series:

$$
\begin{aligned}
E_{\mu \nu}(k)= & E_{\mu \nu}(x P)+\left.\frac{\partial E_{\mu \nu}(k)}{\partial k_{\alpha}}\right|_{k=x P}(k-x P)_{\alpha}+\ldots, \\
& (k-x P)_{\alpha} \approx k_{\alpha}^{T} .
\end{aligned}
$$

Then, using the collinear Ward identity (see, [10])

$$
\frac{\partial E_{\mu \nu}(k)}{\partial k^{\rho}}=E_{\mu \rho \nu}(k, k) .
$$


we, finally, arrive at the factorized (in the momentum space) DVCS amplitude which reads

$$
\begin{aligned}
& T_{\mu \nu}^{(1)}+T_{\mu \nu}^{(2)}=\int_{-1}^{1} d x \operatorname{tr}\left[E_{\mu \nu}(x P) \Gamma(x)\right] \\
& +\int_{-1}^{1} d x_{1} d x_{2} \operatorname{tr}\left[E_{\mu \rho \nu}\left(x_{1}, x_{2}\right) \omega^{\rho \rho^{\prime}} \Gamma_{\rho^{\prime}}\left(x_{1}, x_{2}\right)\right]
\end{aligned}
$$

where $\omega^{\rho \rho^{\prime}}=\delta^{\rho \rho^{\prime}}-n^{\rho^{\prime}} P^{\rho}$, and

$$
\begin{aligned}
& \Gamma_{\underline{\alpha \beta}}(x)=-\int_{-\infty}^{\infty} \frac{d \lambda}{2 \pi} e^{i(x+\xi) \lambda}\left\langle p_{2}\left|\psi_{\underline{\alpha}}(\lambda n) \bar{\psi}_{\underline{\beta}}(0)\right| p_{1}\right\rangle, \\
& \Gamma_{\underline{\alpha \beta}}^{\rho^{\prime}}\left(x_{1}, x_{2}\right)=-\int_{-\infty}^{\infty} \frac{d \lambda_{1}}{2 \pi} \frac{d \lambda_{2}}{2 \pi} e^{i\left(x_{1}+\xi\right) \lambda_{1}+i\left(x_{2}-x_{1}\right) \lambda_{2}} \\
& \times\left\langle p_{2}\left|\psi_{\underline{\alpha}}\left(\lambda_{1} n\right) i \stackrel{\leftrightarrow}{i D^{\rho^{\prime}}}\left(\lambda_{2} n\right) \bar{\psi}_{\underline{\underline{\beta}}}(0)\right| p_{1}\right\rangle,
\end{aligned}
$$

where $i \vec{D}^{\mu}=i \vec{\partial}^{\mu}+g A^{\mu}$ is the QCD covariant derivative in the fundamental representation. This amplitude is also needed to be "factorized" in the Dirac space. This can be reached by making use of the Fierz decomposition over spinor indices.

In fact, the contributions of $T_{\mu \nu}^{(2)}$ are not completely independent from one another because of the QCD equations of motion (e.o.m.) for fermions. The next step is to use the QCD e.o.m. in order to reexpress the contributions of the correlators with the covariant derivative through the correlators which include only $\bar{\psi}$ and $\psi$ fields. Afterwards, the contribution of $T_{\mu \nu}^{(2)}$ presented in terms of the two-particle correlators should be combined together with the contribution of $T_{\mu \nu}^{(1)}$ in order to get the gauge invariant DVCS amplitude at the Born level.

Let us now focus on the QCD equations of motion. For the sake of simplicity, we start within the approximation where the three-particle correlators are absent. Indeed, to derive the gauge invariant amplitude, it is sufficient to consider only the kinematical twist contributions since the kinematical and dynamical twists enter in the QCD e.o.m. and the DVCS amplitude additively (see [11, 16, 21, 22]).

Let us consider the e.o.m. in the following form:

$$
\langle(\overrightarrow{i \hat{\partial}} \psi(z)) \bar{\psi}(0)\rangle=0, \quad\langle\psi(z)(\bar{\psi}(0) \overleftarrow{i \hat{\partial}})\rangle=0
$$

where the Dirac spinor indices are omitted for simplicity.

We want to adapt our approach and use a parametrization-free formalism, according to [12]. To this end, we introduce the notations (here, $\Gamma$ denotes different combinations of $\gamma$-matrices),

$$
\begin{aligned}
& \left\langle p_{2}|\bar{\psi}(0) \Gamma \psi(z)| p_{1}\right\rangle \stackrel{F}{=} \mathcal{F}^{[\Gamma]}(x), \\
& \left\langle p_{2}\left|\bar{\psi}(0) \Gamma i \stackrel{\leftrightarrow}{\partial_{\alpha}^{T}} \psi(z)\right| p_{1}\right\rangle \stackrel{F}{=} \mathcal{F}_{\alpha}^{\left[\stackrel{\leftrightarrow}{\partial^{T}} \Gamma\right]}(x),
\end{aligned}
$$

where $\stackrel{F}{=}$ denotes the Fourier transformation with the measure $\left(z=\lambda n, z^{\prime}=0\right)$

$$
d x e^{-i\left(x P-\frac{\Delta}{2}\right) z+i\left(x P+\frac{\Delta}{2}\right) z^{\prime}} .
$$

In Eq. (14), if $\Gamma$ becomes the $\gamma$-matrix with an open Lorentz index, the functions $\mathcal{F}^{[\Gamma]}(x)$ and $\mathcal{F}_{\alpha}^{\left[{\overleftrightarrow{\partial^{T}}}^{2}\right]}(x)$ should be written with additional Lorentz indices. 
Keeping the vector and axial-vector projections in the Fierz decomposition of Eq. (13) (all other structures do not contribute in the massless quark case), the e.o.m., in terms of the functions (14), take the following form

$$
\begin{aligned}
& \gamma_{T}^{\alpha} \gamma^{-}\left\{\mathcal{F}_{\alpha}^{\left[\partial^{T}\right.} \gamma^{+}\right](x)-x P^{+} \mathcal{F}_{\alpha}^{\left[\gamma_{T}\right]}(x) \\
& \left.+\frac{i}{2} \varepsilon^{\Delta^{T}-\alpha+} \mathcal{F}^{\left[\gamma^{+} \gamma_{5}\right]}(x)+\xi P^{+} i \varepsilon^{\beta-\alpha+} \mathcal{F}_{\beta}^{\left[\gamma_{T} \gamma_{5}\right]}(x)\right\}=0, \\
& \gamma_{T}^{\alpha} \gamma^{-}\left\{i \varepsilon^{\beta-\alpha+} \mathcal{F}_{\beta}^{\left[\stackrel{\leftrightarrow}{T}^{T} \gamma^{+} \gamma_{5}\right]}(x)+\frac{\Delta_{\alpha}^{T}}{2} \mathcal{F}^{\left[\gamma^{+}\right]}(x)\right. \\
& \left.-x P^{+} i \varepsilon^{\beta-\alpha+} \mathcal{F}_{\beta}^{\left[\gamma_{T} \gamma_{5}\right]}(x)+\xi P^{+} \mathcal{F}_{\alpha}^{\left[\gamma_{T}\right]}(x)\right\}=0 .
\end{aligned}
$$

Following [1] and using Eq. (14), the DVCS amplitudes (see, Eq. (11)) can be written as

$$
\begin{aligned}
& T_{\mu \nu}^{(1)}=\int d x \operatorname{tr}\left[\gamma_{\nu} \frac{x \hat{P}+\hat{\bar{Q}}}{(x P+\bar{Q})^{2}} \gamma_{\mu} \gamma^{-}\right] \mathcal{F}^{\left[\gamma^{+}\right]}(x)+ \\
& \int d x \operatorname{tr}\left[\gamma_{\nu} \frac{x \hat{P}+\hat{\bar{Q}}}{(x P+\bar{Q})^{2}} \gamma_{\mu} \gamma_{\alpha}^{T}\right] \mathcal{F}_{\alpha}^{\left[\gamma_{T}\right]}(x)- \\
& \int d x \operatorname{tr}\left[\gamma_{\nu} \frac{x \hat{P}+\hat{\bar{Q}}}{(x P+\bar{Q})^{2}} \gamma_{\mu} \gamma^{-} \gamma_{5}\right] \mathcal{F}^{\left[\gamma^{+} \gamma_{5}\right]}(x)- \\
& \int d x \operatorname{tr}\left[\gamma_{\nu} \frac{x \hat{P}+\overline{\bar{Q}}}{(x P+\bar{Q})^{2}} \gamma_{\mu} \gamma_{\alpha}^{T} \gamma_{5}\right] \mathcal{F}_{\alpha}^{\left[\gamma_{T} \gamma_{5}\right]}(x) \\
& + \text { "crossed", }
\end{aligned}
$$

and

$$
\begin{aligned}
& \left.T_{\mu \nu}^{(2)}=-\int d x \mathcal{F}_{\alpha}^{\left[\partial^{T}\right.} \gamma^{+}\right](x) \\
& \times \operatorname{tr}\left[\gamma_{\nu} \frac{x \hat{P}+\hat{\bar{Q}}}{(x P+\bar{Q})^{2}} \gamma_{\alpha}^{T} \frac{x \hat{P}+\hat{\bar{Q}}}{(x P+\bar{Q})^{2}} \gamma_{\mu} \gamma^{-}\right]+ \\
& \left.\int d x \mathcal{F}_{\alpha}^{\left[\hat{\partial}^{T}\right.} \gamma^{+} \gamma_{5}\right](x) \\
& \times \operatorname{tr}\left[\gamma_{\nu} \frac{x \hat{P}+\hat{\bar{Q}}}{(x P+\bar{Q})^{2}} \gamma_{\alpha}^{T} \frac{x \hat{P}+\hat{\bar{Q}}}{(x P+\bar{Q})^{2}} \gamma_{\mu} \gamma^{-} \gamma_{5}\right] \\
& + \text { "crossed". }
\end{aligned}
$$

As was mentioned above, we now have to use the QCD e.o.m., written in the form of Eqs. (16) and (17), for the amplitude $T_{\mu \nu}^{(2)}$, and then, combining it with $T_{\mu \nu}^{(1)}$, to collect all the similar terms in the final expression. Due to the specific structure of the e.o.m., the correlators with transverse derivatives in $T_{\mu \nu}^{(2)}$ can be eliminated, and can be re-expressed through known correlators without derivatives (this will also be valid for the case with the dynamical twist contributions included). So, one gets

$$
T_{\mu \nu}^{(1)+(2)}=\frac{1}{2 P \cdot q} \int d x\left(\frac{1}{x-\xi+i \epsilon}+\frac{1}{x+\xi-i \epsilon}\right) \mathcal{T}_{\mu \nu}
$$


where

$$
\begin{aligned}
& \mathcal{T}_{\mu \nu}=\left[\xi\left(\delta_{+}^{\nu} P_{\mu}+\delta_{+}^{\mu} P_{\nu}\right)+\delta_{+}^{\mu} \bar{Q}_{\nu}+\delta_{+}^{\nu} \bar{Q}_{\mu}-g_{\mu \nu} \bar{Q}^{-}\right. \\
& \left.+\frac{1}{2} \delta_{+}^{\mu} \Delta_{\nu}^{T}-\frac{1}{2} \delta_{+}^{\nu} \Delta_{\mu}^{T}\right] \mathcal{F}^{\left[\gamma^{+}\right]}(x) \\
& +\left[3 \xi P_{\mu} g_{\nu \alpha}^{T}+\xi P_{\nu} g_{\mu \alpha}^{T}+\bar{Q}_{\mu} g_{\nu \alpha}^{T}+\bar{Q}_{\nu} g_{\mu \alpha}^{T}\right] \mathcal{F}_{\alpha}^{\left[\gamma_{T}\right]}(x) \\
& +i \frac{\xi}{x}\left[\Delta_{\beta}^{T} \delta_{+}^{\nu}-\bar{Q}^{-} g_{\beta \nu}^{T}\right] \varepsilon^{\beta-\mu+} \mathcal{F}^{\left[\gamma^{+} \gamma_{5}\right]}(x) \\
& \left.+i \frac{\xi}{x}\left[-3 \xi P^{\mu} g_{\beta \nu}^{T}+\xi P^{\nu} g_{\beta \mu}^{T}+\bar{Q}^{\nu} g_{\mu \beta}^{T}-\bar{Q}^{\mu} g_{\nu \beta}^{T}\right)\right] \\
& \times \varepsilon^{\alpha-\beta+} \mathcal{F}_{\alpha}^{\left[\gamma_{T} \gamma_{5}\right]}(x),
\end{aligned}
$$

where $\delta_{+}^{\nu}$ denotes the usual Kronecker symbol.

We have thus derived the gauge invariant DVCS amplitude for the most general case of a target with an arbitrary spin which totally coincides with the results obtained in [12] by a different approach. In the present paper, the corrections of the order $O\left(\Delta_{T}^{2} / Q^{2}\right)$ have been neglected. We postpone the discussion of these corrections until a forthcoming analysis. The detailed study of these contributions has recently been presented in [19, 20].

If one now specifies spin of the hadron and, then, uses explicit parameterizations for the corresponding matrix elements, it will, in particular, reproduce the known cases of spin-0 and spin-1/2 (see, [11], [12] - 17]).

We now study DVCS off a spin-1 particle, which is of phenomenological interest in the deuteron case [6, 23]. To this end, we first specify the parametrization of the relevant matrix elements. Namely, the parameterizations for the vector correlators at the leading twist-2 level are (see, [6])

$$
\begin{aligned}
& \left\langle p_{2}, \lambda_{2}\left|\left[\bar{\psi}(0) \gamma_{\mu} \psi(z)\right]^{\mathrm{tw}-2}\right| p_{1}, \lambda_{1}\right\rangle \stackrel{F}{=} \mathcal{F}_{\mu}^{\left[\gamma^{+}\right]}(x)= \\
& e_{2 \alpha}^{*} \mathcal{V}_{\alpha \beta, \mu}^{(i), L}\left(n^{\star}, n, \Delta_{T}\right) e_{1 \beta} H_{i}^{V}(x, \xi),
\end{aligned}
$$

where

$$
\begin{aligned}
& e_{2 \alpha}^{*} \mathcal{V}_{\alpha \beta, \mu}^{(i), L}\left(n^{\star}, n, \Delta_{T}\right) e_{1 \beta} H_{i}^{V}(x, \xi)= \\
& P_{\mu} H_{1, ., 5}^{V}\left(e_{2}^{*}, e_{1} ; x, \xi\right) \equiv P_{\mu}\left\{\left(e_{2}^{*} \cdot e_{1}\right) H_{1}^{V}(x, \xi)\right. \\
& +\left(e_{2}^{*} \cdot P\right)\left(e_{1} \cdot n\right) H_{2}^{V}(x, \xi)+\left(e_{2}^{*} \cdot n\right)\left(e_{1} \cdot P\right) H_{3}^{V}(x, \xi) \\
& +\frac{1}{M^{2}}\left(e_{2}^{*} \cdot P\right)\left(e_{1} \cdot P\right) H_{4}^{V}(x, \xi) \\
& \left.+M^{2}\left(e_{2}^{*} \cdot n\right)\left(e_{1} \cdot n\right) H_{5}^{V}(x, \xi)\right\} .
\end{aligned}
$$

Here, for the sake of conciseness, a new compact notation $H_{1, . .5}^{V}\left(e_{2}^{*}, e_{1} ; x, \xi\right)$ has been introduced. Now, we are in a position to discuss the twist-3 operator matrix elements and their parameterizations. For the vector quark correlator we have

$$
\begin{aligned}
& \left\langle p_{2}, \lambda_{2}\left|\left[\bar{\psi}(0) \gamma_{\mu} \psi(z)\right]^{\mathrm{tw}-3}\right| p_{1}, \lambda_{1}\right\rangle \stackrel{F}{=} \mathcal{F}_{\mu}^{\left[\gamma_{T}\right]}(x)= \\
& e_{2 \alpha}^{*} \mathcal{V}_{\alpha \beta, \mu}^{(i) T}\left(n^{\star}, n, \Delta_{T}\right) e_{1 \beta} G_{i}^{V}(x, \xi),
\end{aligned}
$$

where

$$
\begin{aligned}
& e_{2 \alpha}^{*} \mathcal{V}_{\alpha \beta, \mu}^{(i) T}\left(n^{\star}, n, \Delta_{T}\right) e_{1 \beta} G_{i}^{V}(x, \xi)= \\
& \Delta_{\mu}^{T} G_{1, . ., 5}^{V}\left(e_{2}^{*}, e_{1} ; x, \xi\right)+e_{2 \mu}^{* T}\left(e_{1} \cdot P\right) G_{6}^{V}(x, \xi) \\
& +e_{1 \mu}^{T}\left(e_{2}^{*} \cdot P\right) G_{7}^{V}(x, \xi)+M^{2} e_{2 \mu}^{* T}\left(e_{1} \cdot n\right) G_{8}^{V}(x, \xi) \\
& +M^{2} e_{1 \mu}^{T}\left(e_{2}^{*} \cdot n\right) G_{9}^{V}(x, \xi)
\end{aligned}
$$


Our next step is the parametrization of the axial-vector correlator. In contrast to the vector projection, the Schouten identity plays a crucial role in the determination of the Lorentz independent structures. The twist-2 axial-vector correlator can be parameterized by

$$
\begin{aligned}
& \left\langle p_{2}, \lambda_{2}\left|\left[\bar{\psi}(0) \gamma_{\mu} \gamma_{5} \psi(z)\right]^{\mathrm{tw}-2}\right| p_{1}, \lambda_{1}\right\rangle \stackrel{F}{=} \mathcal{F}_{\mu}^{\left[\gamma^{+} \gamma_{5}\right]}(x)= \\
& -i e_{2 \alpha}^{*} \mathcal{A}_{\alpha \beta, \mu}^{(i), L}\left(n^{\star}, n, \Delta_{T}\right) e_{1 \beta} H_{i}^{A}(x, \xi),
\end{aligned}
$$

where

$$
\begin{aligned}
& e_{2 \alpha}^{*} \mathcal{A}_{\alpha \beta, \mu}^{(i), L}\left(n^{\star}, n, \Delta_{T}\right) e_{1 \beta} H_{i}^{A}(x, \xi)= \\
& \varepsilon_{\mu P e_{2}^{*} e_{1}^{T}} H_{1}^{A}(x, \xi)+\frac{1}{M^{2}} \varepsilon_{\mu P \Delta^{T} e_{2}^{* T}}\left(e_{1} \cdot P\right) H_{2}^{A}(x, \xi) \\
& +\frac{1}{M^{2}} \varepsilon_{\mu P \Delta^{T} e_{1}^{T}}\left(e_{2}^{*} \cdot P\right) H_{3}^{A}(x, \xi) \\
& +\varepsilon_{\mu P \Delta^{T} e_{2}^{* T}}\left(e_{1} \cdot n\right) H_{4}^{A}(x, \xi) .
\end{aligned}
$$

Next, let us consider the twist-3 correlators. Using the light-cone basis, we have fifteen different possible tensors. As in the twist- 2 case, the use of the Schouten identity reduces the number of independent tensors. Indeed, instead of fifteen possible structures we have nine independent tensors which parameterize the twist-3 axial-vector correlators. Finally, the axial-vector correlator reads

$$
\begin{aligned}
& \left\langle p_{2}, \lambda_{2}\left|\left[\bar{\psi}(0) \gamma_{\mu} \gamma_{5} \psi(z)\right]^{\mathrm{tw}-3}\right| p_{1}, \lambda_{1}\right\rangle \stackrel{F}{=} \mathcal{F}_{\mu}^{\left[\gamma_{T} \gamma_{5}\right]}(x)= \\
& -i e_{2 \alpha}^{*} \mathcal{A}_{\alpha \beta, \mu}^{(i) T}\left(n^{\star}, n, \Delta_{T}\right) e_{1 \beta} G_{i}^{A}(x, \xi),
\end{aligned}
$$

where

$$
\begin{aligned}
& e_{2 \alpha}^{*} \mathcal{A}_{\alpha \beta, \mu}^{(i) T}\left(n^{\star}, n, \Delta_{T}\right) e_{1 \beta} G_{i}^{A}(x, \xi)= \\
& \varepsilon_{\mu n P e_{1}^{T}}\left(e_{2}^{*} \cdot P\right) G_{1}^{A}(x, \xi)+\varepsilon_{\mu n P e_{2}^{* T}}\left(e_{1} \cdot P\right) G_{2}^{A}(x, \xi) \\
& +M^{2} \varepsilon_{\mu n P e_{1}^{T}}\left(e_{2}^{*} \cdot n\right) G_{3}^{A}(x, \xi)+M^{2} \varepsilon_{\mu n P e_{2}^{*} T}\left(e_{1} \cdot n\right) \\
& \times G_{4}^{A}(x, \xi)+\frac{1}{M^{2}} \varepsilon_{\mu \Delta_{T} P e_{2}^{*}}\left(e_{1} \cdot P\right) G_{5}^{A}(x, \xi) \\
& +\varepsilon_{\mu \Delta_{T} P e_{2}^{*}}\left(e_{1} \cdot n\right) G_{6}^{A}(x, \xi)+\varepsilon_{\mu \Delta_{T} P e_{1}}\left(e_{2}^{*} \cdot n\right) \\
& \times G_{7}^{A}(x, \xi)+\varepsilon_{\mu \Delta_{T} n e_{2}^{*}}\left(e_{1} \cdot P\right) G_{8}^{A}(x, \xi) \\
& +M^{2} \varepsilon_{\mu \Delta_{T} n e_{1}}\left(e_{2}^{*} \cdot n\right) G_{9}^{A}(x, \xi) .
\end{aligned}
$$

Inserting the explicit parameterizations (20) - (25) into the amplitude (19), we derive the gauge invariant DVCS amplitude for the case of deuteron target:

$$
\begin{aligned}
& T_{\mu \nu}^{\left(\lambda_{1}, \lambda_{2}\right)}=\frac{1}{2 P \cdot \bar{Q}} \int d x \frac{1}{x-\xi+i \epsilon} \\
& \times\left(\mathcal{T}_{\mu \nu}^{(1)}+\mathcal{T}_{\mu \nu}^{(2)}+\mathcal{T}_{\mu \nu}^{(3)}+\mathcal{T}_{\mu \nu}^{(4)}\right)^{\left(\lambda_{1}, \lambda_{2}\right)} \\
& +O\left(\Delta_{T}^{2} ; \bar{M}^{2}\right)+\text { "crossed" }
\end{aligned}
$$


where the structure amplitudes $\mathcal{T}_{\mu \nu}^{(k)}$ read

$$
\begin{aligned}
& \mathcal{T}_{\mu \nu}^{(1)}=H_{1, . ., 4}^{V}\left(x ; e_{1}, e_{2}^{*}\right)\left(2 \xi P_{\mu} P_{\nu}+P_{\mu} \bar{Q}_{\nu}+P_{\nu} \bar{Q}_{\mu}\right. \\
& \left.-g_{\mu \nu}(P \cdot \bar{Q})+\frac{1}{2} P_{\mu} \Delta_{\nu}^{T}-\frac{1}{2} P_{\nu} \Delta_{\mu}^{T}\right)+G_{1, . ., 4}^{V}\left(x ; e_{1}, e_{2}^{*}\right) \\
& \times\left(\xi P_{\nu} \Delta_{\mu}^{T}+3 \xi P_{\mu} \Delta_{\nu}^{T}+\Delta_{\mu}^{T} \bar{Q}_{\nu}+\Delta_{\nu}^{T} \bar{Q}_{\mu}\right) \\
& -\left(\frac{\left(e_{2}^{*} \cdot P\right)\left(e_{1} \cdot P\right)}{M^{2}} G_{5}^{A}(x)+\left(e_{2}^{*} \cdot P\right)\left(e_{1} \cdot n\right) G_{6}^{A}(x)\right. \\
& \left.+\left(e_{1} \cdot P\right)\left(e_{2}^{*} \cdot n\right)\left(G_{7}^{A}(x)-G_{8}^{A}(x)\right)\right) \\
& \times\left(3 \xi P_{\mu} \Delta_{\nu}^{T}-\xi P_{\nu} \Delta_{\mu}^{T}-\Delta_{\mu}^{T} \bar{Q}_{\nu}+\Delta_{\nu}^{T} \bar{Q}_{\mu}\right)
\end{aligned}
$$

and

$$
\begin{aligned}
& \mathcal{T}_{\mu \nu}^{(2)}=\left(e_{1} \cdot P\right) G_{6}^{V}(x)\left(\xi P_{\nu} e_{2 \mu}^{* T}+3 \xi P_{\mu} e_{2 \nu}^{* T}+e_{2 \mu}^{* T} \bar{Q}_{\nu}\right. \\
& \left.+e_{2 \nu}^{* T} \bar{Q}_{\mu}\right)+\left(e_{1} \cdot P\right) G_{2}^{A}(x)\left(3 \xi P_{\mu} e_{2 \nu}^{* T}-\xi P_{\nu} e_{2 \mu}^{* T}\right. \\
& \left.-e_{2 \mu}^{* T} \bar{Q}_{\nu}+e_{2 \nu}^{* T} \bar{Q}_{\mu}\right)
\end{aligned}
$$

and

$$
\begin{aligned}
& \mathcal{T}_{\mu \nu}^{(3)}=\left(e_{2}^{*} \cdot P\right) G_{7}^{V}(x)\left(\xi P_{\nu} e_{1 \mu}^{T}+3 \xi P_{\mu} e_{1 \nu}^{T}+e_{1 \mu}^{T} \bar{Q}_{\nu}\right. \\
& \left.+e_{1 \nu}^{T} \bar{Q}_{\mu}\right)+\left(e_{2}^{*} \cdot P\right) G_{1}^{A}(x)\left(3 \xi P_{\mu} e_{1 \nu}^{T}-\xi P_{\nu} e_{1 \mu}^{T}\right. \\
& \left.-e_{1 \mu}^{T} \bar{Q}_{\nu}+e_{1 \nu}^{T} \bar{Q}_{\mu}\right)
\end{aligned}
$$

and

$$
\begin{aligned}
& \mathcal{T}_{\mu \nu}^{(4)}=\varepsilon_{\mu \nu P n}\left(\varepsilon_{n P e_{2}^{* T} e_{1}^{T}} H_{1}^{A}(x, \xi)\right. \\
& +\frac{1}{M^{2}} \varepsilon_{n P \Delta^{T} e_{2}^{* T}}\left(e_{1} \cdot P\right) H_{2}^{A}(x, \xi) \\
& +\frac{1}{M^{2}} \varepsilon_{n P \Delta^{T}} e_{1}^{T}\left(e_{2}^{*} \cdot P\right) H_{3}^{A}(x, \xi) \\
& \left.+\varepsilon_{n P \Delta^{T} e_{2}^{* T}}\left(e_{1} \cdot n\right) H_{4}^{A}(x, \xi)\right) .
\end{aligned}
$$

This gauge invariant amplitude for DVCS off deuteron is our main result. For the sake of brevity, in Eqs. (27) - (29), we neglected all terms which are proportional to the square of the hadron mass. The full expressions for all amplitudes will be presented in our forthcoming study. 


\section{SINGLE SPIN ASYMMETRY}

In the preceding section, we have obtained the gauge invariant DVCS amplitude which has a significant meaning for the investigation of any observables. As a phenomenologically important example, we now consider the single (electron) spin asymmetry (SSA), which arises in the collision of the longitudinally polarized electron beams with an unpolarized hadron target. The SSA parameter is defined as

$$
\mathcal{A}_{L}=\frac{d \sigma(\rightarrow)-d \sigma(\leftarrow)}{d \sigma(\rightarrow)+d \sigma(\leftarrow)} .
$$

The numerator of Eq. (31) can be expressed through the imaginary part, first, of the interference between the twist-2 and twist-3 helicity DVCS amplitudes and, second, of the interference between the Bethe-Heitler (BH) and DVCS amplitudes. For the JLAB kinematics, the $\left|\mathcal{A}_{\mathrm{DVCS}}\right|^{2}$ contribution can be neglected compared to the interference term because of large contribution of the $\mathrm{BH}$ amplitude.

The DVCS amplitude contributing to exclusive real photon production at $Q^{2} \gg M^{2}$ for the real and virtual photon polarizations, $i$ and $j$, reads

$$
\begin{aligned}
& \mathcal{A}_{\mathrm{DVCS}}^{(i)}=\frac{e_{\ell} e_{q}^{2}}{q^{2}} \sum_{j} L^{(j)} \mathcal{A}_{(j, i)}, \\
& L^{(j)}=\mathcal{L}_{\mu^{\prime}}\left(\ell_{1}, \ell_{2}\right) \epsilon_{\mu^{\prime}}^{*}{ }^{(j)}
\end{aligned}
$$

respectively. Here, the helicity amplitude is given by

$$
\mathcal{A}_{(j, i)}=\epsilon_{\mu}^{(j)} T_{\mu \nu} \epsilon_{\nu}^{\prime *(i)}, \quad i= \pm 1, \quad j=0, \pm 1 .
$$

The Bethe-Heitler amplitude reads

$$
\begin{aligned}
\mathcal{A}_{B H}^{(i)} & =\frac{e_{\ell} e_{q}^{2}}{\Delta^{2}} \sum_{j} \Lambda^{(j, i)} \mathcal{T}_{(j)}, \quad \mathcal{T}_{(j)}=\epsilon_{\mu}^{(j)} F_{\mu}, \\
\Lambda^{(j, i)} & =L_{\mu^{\prime} \nu^{\prime}}\left(\ell_{1}, \ell_{2}\right) \epsilon_{\mu^{\prime}}^{*}{ }^{(j)} \epsilon_{\nu^{\prime}}^{\prime *(i)}
\end{aligned}
$$

where

$$
\Delta^{2}=-4 \xi^{2} \bar{M}^{2}+\Delta_{T}^{2} \equiv t,
$$

where $t$ is negative, For convenience, we introduce the following shorthand notations for Compton form factors related to the various GPDs:

$$
\begin{aligned}
& \int d x \frac{G_{i}^{V}(x, \xi)}{x-\xi+i \epsilon} \Longrightarrow \mathcal{G}_{i}^{V}, \quad \int d x \frac{H_{i}^{V}(x, \xi)}{x-\xi+i \epsilon} \Longrightarrow \mathcal{H}_{i}^{V} \\
& \int d x \frac{\xi}{x} \frac{G_{i}^{A}(x, \xi)}{x-\xi+i \epsilon} \Longrightarrow \mathcal{G}_{i}^{A}
\end{aligned}
$$

and

$$
\begin{aligned}
& \int d x \frac{G_{i}^{V}(x, \xi)}{x-\xi-i \epsilon} \Longrightarrow \overline{\mathcal{G}_{i}^{V}}, \quad \int d x \frac{H_{i}^{V}(x, \xi)}{x-\xi-i \epsilon} \Longrightarrow \overline{\mathcal{H}_{i}^{V}} \\
& \int d x \frac{\xi}{x} \frac{G_{i}^{A}(x, \xi)}{x-\xi-i \epsilon} \Longrightarrow \overline{\mathcal{G}_{i}^{A}}
\end{aligned}
$$

We now calculate the contribution to $\mathcal{A}_{\mathrm{BH}}^{*} \mathcal{A}_{\mathrm{DVCS}}$ coming from the interference between Eqs. (32) and (34). We have the following expressions (here, deuteron polarizations are summed up):

$$
\left.\frac{1}{q^{2} \Delta^{2}} \sum_{i}\left[L^{(0)} \mathcal{A}_{(0, i)}\right] \cdot\left[\Lambda^{(+, i)} \mathcal{T}_{(+)}\right]^{*}\right|_{\mathrm{tw}-2} \sim \frac{1}{\xi(\rho-4) \rho} \sum_{i=1}^{5} \sum_{j=1}^{3} \mathcal{H}_{i}^{V} C_{i j}^{(1)} G_{j}
$$


where

$$
\begin{aligned}
& C_{i j}^{(1)}= \\
& \left(\begin{array}{ccc}
-8\left(\xi^{2}(-4+\rho)-\rho\right)(-4+\rho)(12+(-4+\rho) \rho) & 8\left(\xi^{2}(-4+\rho)-\rho\right)(-4+\rho)(-2+\rho) \rho & -8\left(\xi^{2}(-4+\rho)-\rho\right)(-4+\rho)(-2+\rho) \rho \\
-4\left(\xi^{2}(-4+\rho)-\rho\right)(-4+\rho)(-2+\rho)(\xi(-4+\rho)+\rho) & 4\left(\xi^{2}(-4+\rho)-\rho\right)(-4+\rho) \rho(-2+\xi(-4+\rho)+\rho) & -4\left(\xi^{2}(-4+\rho)-\rho\right)(-4+\rho) \rho(\xi(-4+\rho)+\rho) \\
4(\xi(-4+\rho)-\rho)\left(\xi^{2}(-4+\rho)-\rho\right)(-4+\rho)(-2+\rho) & -4(2+\xi(-4+\rho)-\rho)\left(\xi^{2}(-4+\rho)-\rho\right)(-4+\rho) \rho & 4(\xi(-4+\rho)-\rho)\left(\xi^{2}(-4+\rho)-\rho\right)(-4+\rho) \rho \\
\left(\xi^{2}(-4+\rho)-\rho\right)(-4+\rho)^{2}(-2+\rho) \rho & -\left(\xi^{2}(-4+\rho)-\rho\right)(-4+\rho)^{2} \rho^{2} & \left(\xi^{2}(-4+\rho)-\rho\right)(-4+\rho)^{2} \rho^{2} \\
-16\left(-2+\xi^{2}(-6+\rho)-\rho\right)\left(\xi^{2}(-4+\rho)-\rho\right)(-4+\rho) & 16(-4+\rho)\left(-\xi^{2}(-4+\rho)+\rho\right)^{2} & -16\left(\xi^{4}(-4+\rho)^{3}-2 \xi^{2}(-4+\rho)(-2+\rho) \rho+\rho^{3}\right)
\end{array}\right) \\
& \left.\frac{1}{q^{2} \Delta^{2}} \sum_{i}\left[L^{(0)} \mathcal{A}_{(0, i)}\right] \cdot\left[\Lambda^{(+, i)} \mathcal{T}_{(+)}\right]^{*}\right|_{\mathrm{tw}-3} ^{V} \sim \frac{1}{\xi(\rho-4) \rho}\left(\sum_{i=1}^{9} \sum_{j=1}^{2} \mathcal{G}_{i}^{V} C_{i j}^{(2,1)} G_{j}+\sum_{i=1}^{9} \mathcal{G}_{i}^{V} C_{i}^{(2,2)} G_{3}\right)
\end{aligned}
$$

where

$$
\begin{aligned}
& C_{i j}^{(2,1)}= \\
& \left(\begin{array}{c}
-16 \xi\left(\xi^{2}(-4+\rho)-\rho\right)(-4+\rho)(12+(-4+\rho) \rho) \\
-8 \xi\left(\xi^{2}(-4+\rho)-\rho\right)(-4+\rho)(-2+\rho)(\xi(-4+\rho)+\rho) \\
8 \xi(\xi(-4+\rho)-\rho)\left(\xi^{2}(-4+\rho)-\rho\right)(-4+\rho)(-2+\rho) \\
2 \xi\left(\xi^{2}(-4+\rho)-\rho\right)(-4+\rho)^{2}(-2+\rho) \rho \\
-32 \xi\left(-2+\xi^{2}(-6+\rho)-\rho\right)\left(\xi^{2}(-4+\rho)-\rho\right)(-4+\rho) \\
-4 \xi\left(\xi^{2}(-4+\rho)-\rho\right)(-4+\rho)^{2}(-2+\rho) \\
4 \xi\left(\xi^{2}(-4+\rho)-\rho\right)(-4+\rho)^{2}(-2+\rho) \\
16 \xi\left(\xi^{2}(-4+\rho)-\rho\right)(-4+\rho)(-2+\xi(-6+\rho)+\rho) \\
16 \xi(2+\xi(-6+\rho)-\rho)\left(\xi^{2}(-4+\rho)-\rho\right)(-4+\rho)
\end{array}\right. \\
& 16 \xi\left(\xi^{2}(-4+\rho)-\rho\right)(-4+\rho)(-2+\rho) \rho \\
& 8 \xi\left(\xi^{2}(-4+\rho)-\rho\right)(-4+\rho) \rho(-2+\xi(-4+\rho)+\rho) \\
& -8 \xi(2+\xi(-4+\rho)-\rho)\left(\xi^{2}(-4+\rho)-\rho\right)(-4+\rho) \rho \\
& -2 \xi\left(\xi^{2}(-4+\rho)-\rho\right)(-4+\rho)^{2} \rho^{2} \\
& 32 \xi(-4+\rho)\left(-\xi^{2}(-4+\rho)+\rho\right)^{2} \\
& 4(-1+\xi) \xi(-4+\rho)^{2} \rho(\xi(-4+\rho)+\rho) \\
& -4 \xi(1+\xi)(\xi(-4+\rho)-\rho)(-4+\rho)^{2} \rho \\
& -16 \xi(-4+\rho)\left(\xi^{3}(-4+\rho)^{2}-\xi(-8+\rho) \rho-(-2+\rho) \rho+\xi^{2}(-4+\rho)(2+\rho)\right)
\end{aligned}
$$

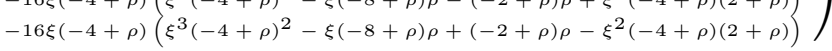

$$
\begin{aligned}
& C_{i}^{(2,2)}=\left(\begin{array}{c}
-16 \xi\left(\xi^{2}(-4+\rho)-\rho\right)(-4+\rho)(-2+\rho) \rho \\
-8 \xi\left(\xi^{2}(-4+\rho)-\rho\right)(-4+\rho) \rho(\xi(-4+\rho)+\rho) \\
8 \xi(\xi(-4+\rho)-\rho)\left(\xi^{2}(-4+\rho)-\rho\right)(-4+\rho) \rho \\
2 \xi\left(\xi^{2}(-4+\rho)-\rho\right)(-4+\rho)^{2} \rho^{2} \\
-32 \xi\left(\xi^{4}(-4+\rho)^{3}-2 \xi^{2}(-4+\rho)(-2+\rho) \rho+\rho^{3}\right) \\
-4 \xi\left(\xi^{2}(-4+\rho)-\rho\right)(-4+\rho)^{2} \rho \\
4 \xi\left(\xi^{2}(-4+\rho)-\rho\right)(-4+\rho)^{2} \rho \\
16 \xi\left(\xi^{2}(-4+\rho)-\rho\right)(-4+\rho)(\xi(-4+\rho)+\rho) \\
16 \xi(\xi(-4+\rho)-\rho)\left(\xi^{2}(-4+\rho)-\rho\right)(-4+\rho)
\end{array}\right), \\
& \left.\frac{1}{q^{2} \Delta^{2}} \sum_{i}\left[L^{(0)} \mathcal{A}_{(0, i)}\right] \cdot\left[\Lambda^{(+, i)} \mathcal{T}_{(+)}\right]^{*}\right|_{\mathrm{tw}-3} ^{A} \sim \frac{1}{\xi(\rho-4) \rho}\left(\sum_{i=1}^{9} \sum_{j=1}^{2} \overline{\mathcal{G}}_{i}^{A} C_{i j}^{(3,1)} G_{j}+\sum_{i=1}^{9} \overline{\mathcal{G}}_{i}^{A} C_{i}^{(3,2)} G_{3}\right)
\end{aligned}
$$

where

$$
\begin{aligned}
& C_{i j}^{(3,1)}= \\
& \left(\begin{array}{cc}
4 \xi\left(\xi^{2}(-4+\rho)-\rho\right)(-4+\rho)^{2}(-2+\rho) & -4 \xi(1+\xi)(\xi(-4+\rho)-\rho)(-4+\rho)^{2} \rho \\
-4 \xi\left(\xi^{2}(-4+\rho)-\rho\right)(-4+\rho)^{2}(-2+\rho) & 4(-1+\xi) \xi(-4+\rho)^{2} \rho(\xi(-4+\rho)+\rho) \\
16 \xi(2+\xi(-6+\rho)-\rho)\left(\xi^{2}(-4+\rho)-\rho\right)(-4+\rho) & -16 \xi(-4+\rho)\left(\xi^{3}(-4+\rho)^{2}-\xi(-8+\rho) \rho+(-2+\rho) \rho-\xi^{2}(-4+\rho)(2+\rho)\right) \\
16 \xi\left(\xi^{2}(-4+\rho)-\rho\right)(-4+\rho)(-2+\xi(-6+\rho)+\rho) & -16 \xi(-4+\rho)\left(\xi^{3}(-4+\rho)^{2}-\xi(-8+\rho) \rho-(-2+\rho) \rho+\xi^{2}(-4+\rho)(2+\rho)\right) \\
-2 \xi\left(\xi^{2}(-4+\rho)-\rho\right)(-4+\rho)^{2}(-2+\rho) \rho & 2 \xi\left(\xi^{2}(-4+\rho)-\rho\right)(-4+\rho)^{2} \rho^{2} \\
8 \xi\left(\xi^{2}(-4+\rho)-\rho\right)(-4+\rho)(-2+\rho)(\xi(-4+\rho)+\rho) & -8 \xi\left(\xi^{2}(-4+\rho)-\rho\right)(-4+\rho) \rho(-2+\xi(-4+\rho)+\rho) \\
-8 \xi(\xi(-4+\rho)-\rho)\left(\xi^{2}(-4+\rho)-\rho\right)(-4+\rho)(-2+\rho) & 8 \xi(2+\xi(-4+\rho)-\rho)\left(\xi^{2}(-4+\rho)-\rho\right)(-4+\rho) \rho \\
8 \xi(\xi(-4+\rho)-\rho)\left(\xi^{2}(-4+\rho)-\rho\right)(-4+\rho)(-2+\rho) & -8 \xi(2+\xi(-4+\rho)-\rho)\left(\xi^{2}(-4+\rho)-\rho\right)(-4+\rho) \rho \\
-32 \xi\left(-2+\xi^{2}(-6+\rho)-\rho\right)\left(\xi^{2}(-4+\rho)-\rho\right)(-4+\rho) & 32 \xi(-4+\rho)\left(-\xi^{2}(-4+\rho)+\rho\right)^{2}
\end{array}\right. \\
& C_{i}^{(3,2)}=\left(\begin{array}{c}
4 \xi\left(\xi^{2}(-4+\rho)-\rho\right)(-4+\rho)^{2} \rho \\
-4 \xi\left(\xi^{2}(-4+\rho)-\rho\right)(-4+\rho)^{2} \rho \\
16 \xi(\xi(-4+\rho)-\rho)\left(\xi^{2}(-4+\rho)-\rho\right)(-4+\rho) \\
16 \xi\left(\xi^{2}(-4+\rho)-\rho\right)(-4+\rho)(\xi(-4+\rho)+\rho) \\
-2 \xi\left(\xi^{2}(-4+\rho)-\rho\right)(-4+\rho)^{2} \rho^{2} \\
8 \xi\left(\xi^{2}(-4+\rho)-\rho\right)(-4+\rho) \rho(\xi(-4+\rho)+\rho) \\
-8 \xi(\xi(-4+\rho)-\rho)\left(\xi^{2}(-4+\rho)-\rho\right)(-4+\rho) \rho \\
8 \xi(\xi(-4+\rho)-\rho)\left(\xi^{2}(-4+\rho)-\rho\right)(-4+\rho) \rho \\
-32 \xi\left(\xi^{4}(-4+\rho)^{3}-2 \xi^{2}(-4+\rho)(-2+\rho) \rho+\rho^{3}\right)
\end{array}\right)
\end{aligned}
$$


Here, $\rho=t / M^{2}$. In the last expression, $G_{1,2,3}$ are the known electromagnetic deuteron form factors.

As it can be seen from the parameterizations introduced above, the only surviving contributions in the forward limit, are related to the Compton form factors $\mathcal{H}_{1,5}$ and $\mathcal{G}_{8,9}$ terms. Keeping only these contributions, one can write

$$
\begin{aligned}
& \frac{1}{q^{2} \Delta^{2}} \sum_{i}\left[L^{(0)} \mathcal{A}_{(0, i)}\right] \cdot\left[\Lambda^{(+, i)} \mathcal{T}_{(+)}\right]^{*} \sim \frac{1}{\xi(\rho-4) \rho} \times \\
& \left\{G _ { 1 } \left(16 \xi\left(\xi^{2}(\rho-4)-\rho\right)(\rho-4)(\xi(\rho-6)+\rho-2) \mathcal{G}_{8}^{V}+16 \xi(\xi(\rho-6)-\rho+2)\left(\xi^{2}(\rho-4)-\rho\right)(\rho-4) \mathcal{G}_{9}^{V}\right.\right. \\
& \left.-8\left(\xi^{2}(\rho-4)-\rho\right)(\rho-4)((\rho-4) \rho+12) \mathcal{H}_{1}^{V}-16\left((\rho-6) \xi^{2}-\rho-2\right)\left(\xi^{2}(\rho-4)-\rho\right)(\rho-4) \mathcal{H}_{5}^{V}\right) \\
& +G_{2}\left(-16 \xi(\rho-4)\left((\rho-4)^{2} \xi^{3}+(\rho-4)(\rho+2) \xi^{2}-(\rho-8) \rho \xi-(\rho-2) \rho\right) \mathcal{G}_{8}^{V}-16 \xi(\rho-4)\left((\rho-4)^{2} \xi^{3}\right.\right. \\
& \left.-(\rho-4)(\rho+2) \xi^{2}-(\rho-8) \rho \xi+(\rho-2) \rho\right) \mathcal{G}_{9}^{V}+8\left(\xi^{2}(\rho-4)-\rho\right) \rho((\rho-6) \rho+8) \mathcal{H}_{1}^{V} \\
& \left.+16(\rho-4)\left(\rho-\xi^{2}(\rho-4)\right)^{2} \mathcal{H}_{5}^{V}\right)+G_{3}\left(16 \xi\left(\xi^{2}(\rho-4)-\rho\right)(\rho-4)(\xi(\rho-4)+\rho) \mathcal{G}_{8}^{V}\right. \\
& +16 \xi(\xi(\rho-4)-\rho)\left(\xi^{2}(\rho-4)-\rho\right)(\rho-4) \mathcal{G}_{9}^{V}-8\left(\xi^{2}(\rho-4)-\rho\right) \rho((\rho-6) \rho+8) \mathcal{H}_{1}^{V}-16\left((\rho-4)^{3} \xi^{4}\right. \\
& \left.\left.\left.-2 \rho((\rho-6) \rho+8) \xi^{2}+\rho^{3}\right) \mathcal{H}_{5}^{V}\right)\right\}+\ldots .
\end{aligned}
$$

If we now calculate the imaginary part of the above-mentioned terms, we will obtain the numerator for experimentally accessible single spin asymmetry parameter [25].

\section{CONCLUSIONS}

In conclusion, we have derived the gauge invariant amplitude for the deeply virtual Compton scattering off a spin-1 hadron. As an important phenomenological application of this approach, we have considered the deuteron target and have presented the gauge invariant DVCS amplitude for the deuteron case. We have also discussed the simplest kind of asymmetries - the single spin asymmetry where the initial lepton has a longitudinal polarization while all other particles, the initial hadron, the final lepton and the final hadron, are unpolarized.

\section{ACKNOWLEDGEMENTS}

The authors would like to thank A.V. Efremov, G. Ingelman, D. Ivanov, L. Szymanowski, S. Wallon for useful discussions and comments. This work is supported in part by the DFG project BR2021/6-1, the RFBR (grants 09-02-01149, 11-02-01454, 12-02-00613) and by the Carl Trygger Foundation (Sweden).

[1] F. Ellinghaus, R. Shanidze and J. Volmer [HERMES Collaboration], AIP Conf. Proc. 675, 303 (2003) arXiv:hep-ex/0212019.

[2] M. Mazouz et al. [Jefferson Lab Hall A Collaboration], Phys. Rev. Lett. 99, 242501 (2007) arXiv:0709.0450 [nucl-ex]].

[3] E. Voutier, arXiv:0809.2670 [nucl-ex].

[4] A. Airapetian et al. [HERMES Collaboration], Nucl. Phys. B 829, 1 (2010) arXiv:0911.0095 [hep-ex]].

[5] F. Cano and B. Pire, Eur. Phys. J. A 19, 423 (2004) arXiv:hep-ph/0307231.

[6] E. R. Berger, F. Cano, M. Diehl and B. Pire, Phys. Rev. Lett. 87, 142302 (2001) arXiv:hep-ph/0106192.

[7] A. Kirchner and D. Mueller, Eur. Phys. J. C 32, 347 (2003)].

[8] P.A.M. Guichon, M. Vanderhaegen, Prog. Part. Phys. 41 (1998) 125 ; X. Ji, J. Phys. G24 (1998) 1181.

[9] R.K. Ellis, W. Furmanski, R. Petronzio, Nucl. Phys. B207,1 (1982).

[10] A.V. Efremov and O.V. Teryaev Yad. Phys. 39, 1517 (1984)

[11] I. V. Anikin, B. Pire and O. V. Teryaev, Phys. Rev. D 62, 071501 (2000).

[12] M. Penttinen, M. V. Polyakov, A. G. Shuvaev and M. Strikman, Phys. Lett. B 491, 96 (2000) arXiv:hep-ph/0006321.

[13] A. V. Belitsky, D. Mueller, L. Niedermeier and A. Schafer, Nucl. Phys. B 593, 289 (2001) arXiv:hep-ph/0004059.

[14] A. V. Belitsky and D. Mueller, Nucl. Phys. B 589, 611 (2000) arXiv:hep-ph/0007031. 
[15] M. Vanderhaeghen, Eur. Phys. J. A 8, 455 (2000) arXiv:hep-ph/0007232.

[16] N. Kivel, M. V. Polyakov, A. Schafer and O. V. Teryaev, Phys. Lett. B 497, 73 (2001) arXiv:hep-ph/0007315.

[17] A. V. Radyushkin and C. Weiss, Phys. Lett. B 493, 332 (2000) arXiv:hep-ph/0008214].

[18] I. V. Anikin, D. Yu. Ivanov, B. Pire, L. Szymanowski and S. Wallon, Nucl. Phys. B 828, 1 (2010) arXiv:0909.4090 [hep-ph]]; I. V. Anikin, D. Yu. Ivanov, B. Pire, L. Szymanowski and S. Wallon, Phys. Lett. B 682, 413 (2010) arXiv:0903.4797 [hep-ph]].

[19] V. M. Braun and A. N. Manashov, Phys. Rev. Lett. 107, 202001 (2011) arXiv:1108.2394 [hep-ph]].

[20] V. M. Braun and A. N. Manashov, JHEP 1201, 085 (2012) [arXiv:1111.6765 [hep-ph]].

[21] I. V. Anikin and O. V. Teryaev, Phys. Lett. B509, 95 (2001)

[22] I. V. Anikin and O. V. Teryaev, Phys. Lett. B554, 51 (2003)

[23] A. Kirchner, D. Mueller, Eur. Phys. J. C32, 347-375 (2003); F. Cano, B. Pire, Eur. Phys. J. A19, 423-438 (2004).

[24] I. V. Anikin, R. S. Pasechnik, B. Pire and O. V. Teryaev, PoSICHEP 2010, 137 (2010) arXiv:1011.2853 [hep-ph]].

[25] A. Airapetian et al. [HERMES Collaboration], Nucl. Phys. B 829, 1 (2010). 